\title{
Particle diffusion on a three-dimensional random sea
}

\author{
J. M. Buick, I. G. Morrison, T. S. Durrani, C. A. Greated
}

\begin{abstract}
The diffusion of particles on a random sea surface, due to the random fluctuations of the Stokes drift velocity, is examined. Experimental results are presented for the diffusion of particle pairs on the surface of a three-dimensional random sea with a Pierson-Moskowitz surface spectrum and a $\cos ^{2}$ spreading function. The results show good agreement with theoretical predictions, particularly at low separations where other affects are negligible. Diffusion of surface particles in different sea states is also discussed.

\section{List of symbols}

$\alpha$

Phillips constant

angle between $r$ and $x$-axis

$\delta(x) \quad$ Dirac delta function

$\lambda_{\mathrm{m}} \quad$ peak wavelength of the spectrum corre-

sponding to the frequency $\omega_{\mathrm{m}}$

$\theta \quad$ angle in $x-y$ plane

$\omega \quad$ angular frequency

$\omega_{\mathrm{m}} \quad$ peak frequency of the frequency spectrum

$D_{i j} \quad$ diffusion tensor

$\bar{D}_{i j} \quad$ non-dimensional part of $D_{i j}$

$D \quad$ value of the best-fit curve through the measured values of $D_{x x}$

$\Delta D \quad$ difference between the measured value of $D_{x x}$ and $D$

$\mathrm{F}(\mathbf{k}) \quad$ two-dimensional wave spectrum
\end{abstract}

Received: 18 March 1999/Accepted: 6 March 2000

J. M. Buick ( $\bowtie)$, T. S. Durrani

Signal Processing Division

Department of Electrical and Electronic Engineering

The University of Strathclyde, Glasgow G1 1XQ, Scotland, UK

I. G. Morrison, C. A. Greated

Department of Physics and Astronomy

The University of Edinburgh, The Kings Buildings

Mayfield Road, Edinburgh EH9 3JZ, Scotland, UK

Present address:

Department of Physics and Astronomy,

The University of Edinburgh, The Kings Buildings

Mayfield Road, Edinburgh EH9 3JZ, Scotland, UK

This work was supported by the Science and Engineering Research Council and the Marine Technology Directorate UK.

$\begin{array}{ll}f(\omega) & \text { surface frequency spectrum } \\ g & \text { acceleration due to gravity } \\ \left\langle h^{2}\right\rangle & \text { mean square surface displacement } \\ \mathbf{k} & \text { two-dimensional wave number } \\ M_{i j}\left(\theta_{1}, \theta_{2}\right) & \text { term in the diffusion tensor } \\ P\left(\theta_{1}, \theta_{2}, \omega\right) & \text { term in the diffusion tensor for a pair of } \\ & \text { particles } \\ \mathbf{r} & \text { separation of particles } \\ \Delta r_{i} & \text { change in } r_{i} \\ S(\theta) & \text { spreading function } \\ U & \text { wind speed } \\ \mathbf{u}_{\mathrm{s}} & \text { Stokes drift velocity } \\ x & \text { horizontal co-ordinate in wind direction } \\ y & \text { orthogonal horizontal co-ordinate } \\ z & \text { vertical co-ordinate }\end{array}$

1

\section{Introduction}

Pollution in the ocean is a significant problem, since it endangers the lives of marine animals such as fish and sea birds, destroys coral reefs and contaminates breaches and coastal regions. The action of pollution in the sea may also adversely affect humans who come into contact with it, either through bathing in the sea or consuming sea food from polluted waters. Pollution can occur in many forms, ranging from large-scale oil spills (which can occur, for example, if an oil tanker runs aground in severe weather or if two tankers collide) to smaller-scale spills (such as a small discharge from a chemical or sewage plant where even a small daily discharge could, depending on the manner in which the pollution is dispersed, build up to form a significantly large contaminated region). Whatever the method of pollution, it is clearly important to have a good understanding of the methods in which the pollutants are dispersed, either in forming a contingency plan in order to react to a major disaster, or predicting the level of pollution close to a permanent source of contamination. There are a number of processes which contribute to the total diffusion, such as turbulence (Maksimenko 1991; Craig and Banner 1994), and ocean currents and tidal flows (Sanderson and Pall 1990), which affect the displacement of particles on a large scale. On a smaller scale of up to a few kilometres, the action of water waves has a large role to play (Schott et al. 1978; Herterich and Hasselman 1982). This has been demonstrated by recent oil spills where the dispersion of pollutants has been seen to be highly dependent on the prevailing wave climate. In many cases, for example when an oil tanker hits rocks close to the coast, the dispersion of the pollution on a 
small scale is of vital importance. It is also important in confined regions such as ports and estuaries, where the typical length scale is no larger than a few kilometres and where there can often be a large number of ships and coastal factories, each contributing to the total pollution. Despite this, the action of wave motion is not normally considered in numerical simulations and models of pollution dispersion.

The diffusion of tracers at the sea surface under the action of random wave motion has been investigated by Herterich and Hasselmann (1982). They study this by considering the random fluctuations in the Stokes drift velocity $\mathbf{u}_{\mathrm{s}}$ which occur due to the random nature of the surface motion. The Stokes drift velocity arises from the particle trajectories of progressive waves not being completely closed and for deep-water, sinusoidal, progressive waves in a random sea the Stokes drift velocity is given by $\left\langle\mathbf{u}_{\mathrm{s}}\right\rangle=2 \int F(\mathbf{k}) \omega \mathbf{k e}^{2 k z} \mathrm{~d}^{2} k$, where angle brackets denote ensemble averaging, $z$ is the vertical co-ordinate measured positively upward from the mean surface, $\mathbf{k}$ is the twodimensional wave number in the $x-y$ plane, $\omega=\sqrt{g k}$ is the angular frequency, $g$ is the gravitational acceleration, and the two-dimensional wave spectrum $F(\mathbf{k})$ is normalised to the mean-square surface displacement $\left\langle h^{2}\right\rangle$ : $\int F(\mathbf{k}) \mathrm{d}^{2} k=\left\langle h^{2}\right\rangle$. Herterich and Hasselmann (1982) show that whenever the surface spectrum can be expressed as the product of a frequency spectrum, $f(\omega)$, and a spreading function, $S(\theta)$, the dispersion of particles is governed by a mean advection velocity, the mean Stokes drift velocity, and a diffusion tensor $D_{i j}$ which is associated with the random wave surface. They consider the form of the diffusion tensor for a pair of particles separated by a vector $\mathbf{r}$ at an angle $\beta$ to the $x$ axis (the wind direction), which is given by

$$
\begin{aligned}
& \left(\begin{array}{ll}
D_{x x} & D_{x y} \\
D_{y x} & D_{y y}
\end{array}\right) \\
& =\frac{\pi}{4 g^{2}} \int_{0}^{\infty} \omega^{6} f^{2}(\omega) \int_{-\pi}^{\pi} \int_{-\pi}^{\pi} S\left(\theta_{1}\right) S\left(\theta_{2}\right) P\left(\theta_{1}, \theta_{2}, \omega\right) \\
& \times M_{i j}\left(\theta_{1}, \theta_{2}\right) \mathrm{d} \theta_{1} \mathrm{~d} \theta_{2} \mathrm{~d} \omega
\end{aligned}
$$

where $P\left(\theta_{1}, \theta_{2}, \omega\right)=2\left(1-\cos \left\{\omega^{2} r\left[\cos \left(\theta_{1}-\beta\right)\right.\right.\right.$ $\left.\left.\left.-\cos \left(\theta_{2}-\beta\right)\right] / g\right\}\right)\left[1+\cos \left(\theta_{1}-\theta_{2}\right)\right]^{2}$ and, provided $S(\theta)$ is symmetric about the wind direction $\theta=0$, $D_{x y}=D_{y x}=0$ and $M_{x x}\left(\theta_{1}, \theta_{2}\right)=\left(\cos \theta_{1}+\cos \theta_{2}\right)^{2}$ and $M_{y y}\left(\theta_{1}, \theta_{2}\right)=\left(\sin \theta_{1}+\sin \theta_{2}\right)^{2}$. A similar approach has been used to consider the diffusion due to internal waves (Sanderson and Okubo 1988) and capillary waves (Mesquita et al. 1992).

In this paper, we present some experimental results verifying the theoretical predictions of Herterich and Hasslemann (1982) for the diffusion of a pair of particles on the surface of a three-dimensional random sea.

\section{2}

\section{Experimental set-up}

The object here is to measure the diffusion of marker particles on the surface of a random sea for a precisely defined three-dimensional sea state. The experiments were conducted in the three-dimensional random sea basin at the University of Edinburgh. This is $27.5 \mathrm{~m}$ long $12 \mathrm{~m}$ wide with a water depth of $1.2 \mathrm{~m}$. The tank was built in 1977 and was designed for research at length ratios of between 1:150 and 1:100 in order to accurately represent Atlantic wave activity in a region with length up to $3 \mathrm{~m}$ full scale. One length of the tank has a row of wavemaker paddles. A plastic membrane forms a seal between the water and the wavemakers, so that the wave motion is produced only at the front surface of the wavemaker. This prevents the generation of waves at the rear of the paddle which could resonate in the confined area and degrade the quality of the desired waves. Each wavemaker is fitted with a feedback loop which allows the paddle to also act to absorb any reflected wave which is incident on it. The feedback loop also linearises the response of the wavemaker. The opposite length of the basin and one of the widths are fitted with an absorbent beach. These consist of 2-m-long cages filled with expanded aluminium foil. This proves very efficient in absorbing the wave energy. The other wall of the basin is rigid and partially glazed to allow an underwater view of the motion. This gives a total working area of about $25 \times 5 \mathrm{~m}$, the pitch of the wavemakers is $305 \mathrm{~mm}$. The 75 driven wavemakers are computer controlled to produce waves of different amplitudes, frequencies and angle of travel (relative to the line of paddles). Standard wave spectra, such as the Pierson-Moskowitz and JONSWAP spectra, are built in as high-level functions allowing the operator to re-create these spectra with a spreading function of the form $S(\theta)=\cos ^{2 n} \theta$.

The most obvious parameter to measure in practical terms is the relative diffusion of marked particle pairs. The dispersion rate can then be related to the dispersion of a single particle or to the spread of an oil patch in the sea (Herterich and Hasselmann 1982). The main aim is to verify experimentally the theoretical result (Herterich and Hasselmann 1982) for the diffusion of a pair of particles. The motion of single particles is dominated by the oscillatory motion of the waves, and the diffusion rates can only be measured by tracking them over very long periods and measuring their displacement relative to the mean Stokes drift, which is not possible in a laboratory basin.

Small buoyant particles were made by coating discs of paper with varnish, Clusters of these were spread onto the water surface, and their motion was recorded by imaging them with a CCD camera mounted on a gantry $4 \mathrm{~m}$ above the surface. Particle pairs were then located and their coordinates were recorded in successive frames. From these co-ordinate values, the time histories of the separation in the mean wave direction and the orthogonal direction were recorded. The records were then converted into diffusion coefficients. A schematic representation of the experimental set-up is shown in Fig. 1.

\section{3}

\section{Results}

The Pierson-Moskowitz spectrum (Pierson and Moskowitz 1964) has the form 


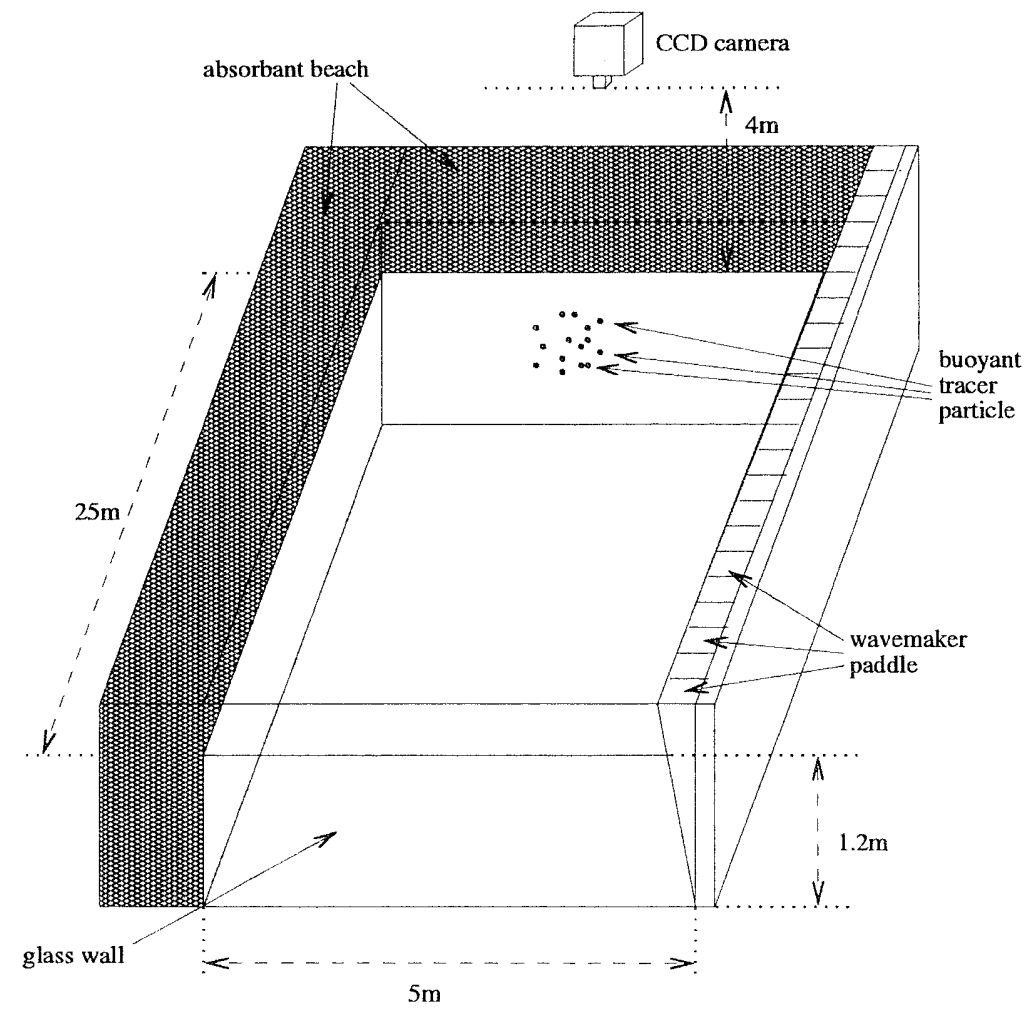

Fig. 1. Schematic diagram of the three-dimensional wave basin and the experimental set-up $f(\omega)=0.0081 g^{2} \omega^{-5} \exp \left[-\frac{5}{4}\left(\frac{\omega_{\mathrm{m}}}{\omega}\right)^{4}\right]$,

where $\omega_{\mathrm{m}}$ is the peak frequency. It represented a fully developed sea state and is the frequency spectrum which was used in the experiments in conjunction with the normalised spreading function $S(\theta)=2 \cos ^{2}(\theta) / \pi$.

The experiments were performed assuming that the basin is a true representation of the ocean at a length scale of 1:100. Then, since $g$ is unscaled between the ocean and the wave tank, the tank represents a $1: \sqrt{100}$ or $1: 10$ time scale of the ocean, because the wave frequency and wave number are related through the dispersion relation

$\omega^{2}=g k$.

The surface spectrum was produced using the array of wave paddles. To compare the wave spectrum produced by the paddles with the desired Pierson-Moskowitz spectrum, a wave gauge was used to measure the surface displacement. This was done using a two-dimensional spectrum with $S(\theta)=\delta(\theta)$, the Dirac delta function. The wave gauge consists of two parallel metal rods which are partially immersed in the water. The gauge measures the conduction of the water between the rods and gives an output voltage which is a linear function of the immersion depth. It is found that the gauge can give an accurate measure of the surface displacement for disturbances greater than $1 \mathrm{~mm}$. Figure 2 shows the Fourier transform of the surface profile and the Pierson-Moskowitz spectrum plotted at ocean scale. The spectrum generated in the tank agrees well with the Pierson-Moskowitz spectrum, although at higher frequencies the spectrum is cut off due to the limitations of the wavemaker.

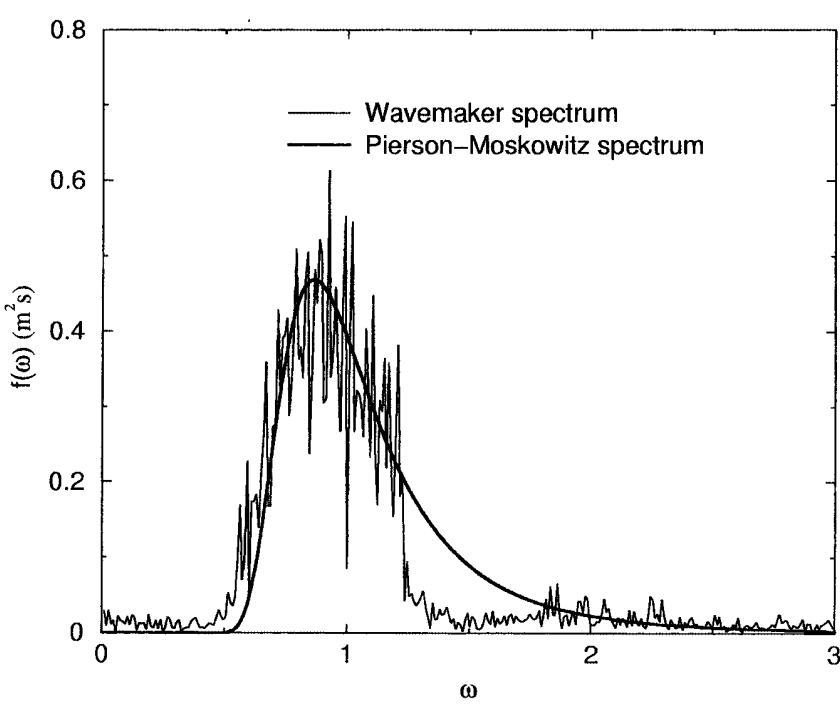

Fig. 2. The surface spectrum calculated by Fourier transforming the surface displacement, plotted at ocean scale. The PiersonMoskowitz spectrum, [Eq. (2)] is also shown for comparison

The components of the diffusion tensor were calculated from the measured components of the particle pair separation, $\mathbf{r}=\left(r_{x}, r_{y}\right)$. The diffusion is given by

$2 D_{i i} t=\left(\Delta r_{i}\right)\left(\Delta r_{i}\right)$,

where $\Delta r_{i}$ is the change in $r_{i}$. Differentiating with respect to $t$, we can write

$D_{i i} \simeq r_{i} \frac{\mathrm{d} r_{i}}{\mathrm{~d} t}$. 
This expression was used to calculate $D_{i i}$ where the derivative was found as a forward difference.

The peak frequency of the Pierson-Moskowitz spectrum, $\omega_{\mathrm{m}}$, is related to the wind speed by

$\omega_{\mathrm{m}}=0.140 \frac{2 \pi g}{U}$

where $U$ is the wind speed measured $19.5 \mathrm{~m}$ above the sea height. To represent a wind speed of $10 \mathrm{~m} \mathrm{~s}^{-1}$ at the ocean scale we set $U=1 \mathrm{~m} \mathrm{~s}^{-1}$ in Eq. (6) to calculate the value of $\omega_{\mathrm{m}}$ for the wave spectrum used in the basin.

The region of interest is the diffusion at scales less than the mean wavelength, that is a few tens of metres (ocean scale). For larger times, it is known that the diffusion rate increases, reaching an asymptotic situation where the mean square separation increases in proportion to time. Measured diffusion coefficients are plotted (at ocean scale) in Figs. 3 and 4 and compared with the theoretical predictions. It can be seen that the experimental results are in good agreement with the theory, particularly at the smallest separations. At larger separations, the measured diffusion tends to be slightly greater than the theoretical value. This is probably due to the fact that turbulent diffusion is starting to have a significant effect. The diffusion rate was observed to be anisotropic, the particles moving more rapidly in the direction of the wave propagation than in the transverse direction. The anisotropy is to be expected and is in agreement with the theoretical predictions.

Since we are dealing with a random process, we do not expect different particle pairs to be diffused by the same amount, rather we expect their diffusion to vary about a mean value. Comparing Eq. (4) with the theoretical definition of $D_{i j}$ (Herterich and Hasselmann 1982), we see that the theory corresponds to the mean diffusion rates, while the experimental results presented here will vary about their mean value. The spread of results is clearly visible

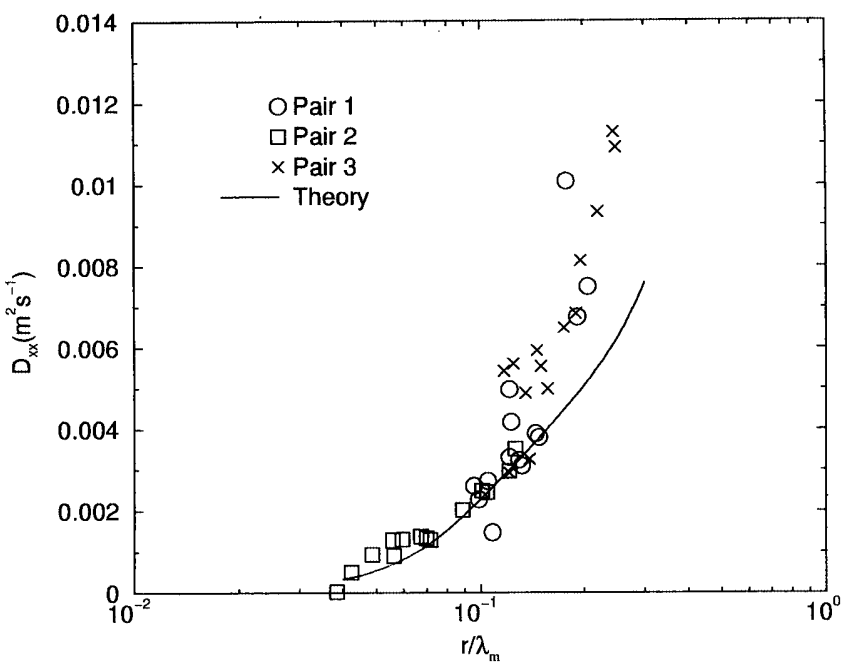

Fig. 3. Measured and theoretical values of the surface diffusion coefficient $D_{x x}$ for particle pairs in a three-dimensional random sea. The surface spectrum is a Pierson-Moskowitz spectrum corresponding to a wind speed of $10 \mathrm{~m} \mathrm{~s}^{-1}$ with a $\cos ^{2}$ spreading function

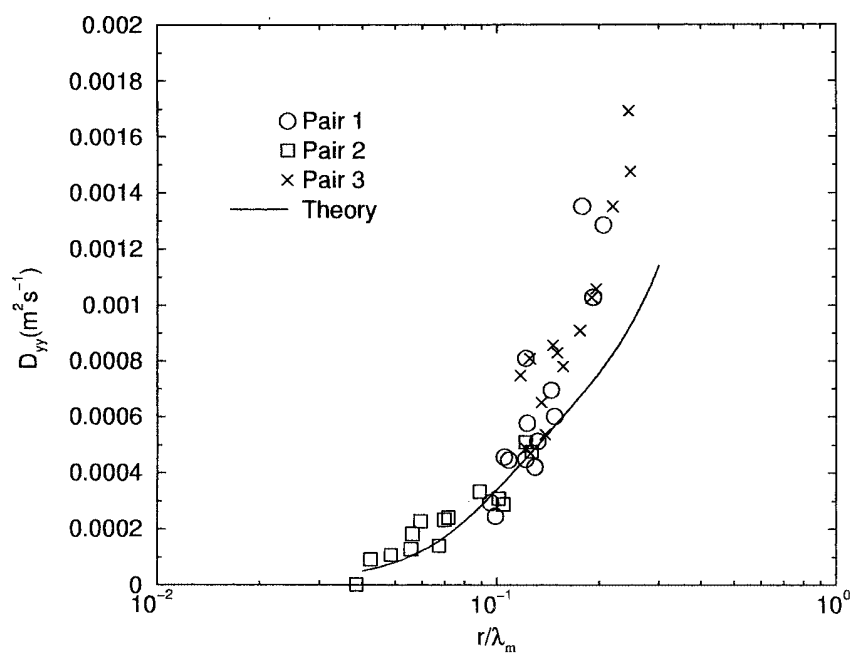

Fig. 4. Measured and theoretical values of the surface diffusion coefficient $D_{y y}$ for particle pairs in a three-dimensional random sea. The surface spectrum is a Pierson-Moskowitz spectrum corresponding to a wind speed of $10 \mathrm{~m} \mathrm{~s}^{-1}$ with a $\cos ^{2}$ spreading function

in Figs. 3 and 4 and is due to the random nature of the waves. A best-fit third-order polynomial was drawn through the results for $D_{x x}$ and, for each point, the deviation between the measured diffusion and the curve was obtained. This deviation, $\Delta D$, is shown in Fig. 5, normalised by the value of the best-fit curve, $D$, in the form of a histogram. This shows the relative variation in the results from their mean value. Also shown in Fig. 5 is the best-fit Gaussian, which has a half width of 0.22 (or $22 \%$ ). This shows that the variation in the results about their mean is approximately Gaussian, as would be expected, and that the half width of the Gaussian is proportional to the magnitude of the diffusion tensor. This is consistent with the random nature of the motion. Note that we have used a

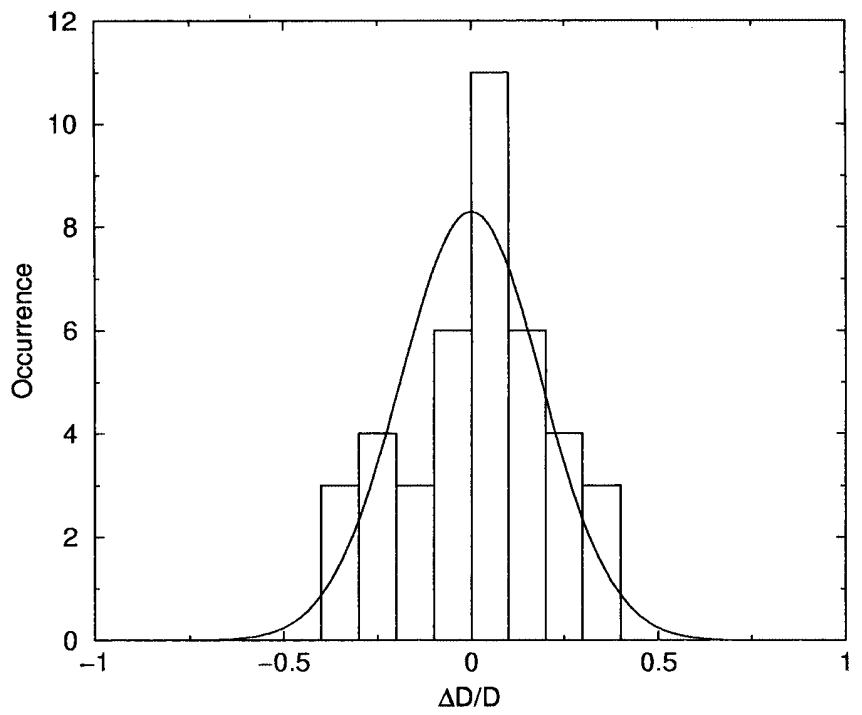

Fig. 5. The deviation, $\Delta D$, between the measured diffusion and a best-fit cure, normalised by the value of the best-fit curve, $D$, is shown in the form of a histogram, Also shown is the best fit Gaussian curve 
best-fit curve here, rather than the theory, because of the differences between the theory and the measurements.

\section{4}

\section{Discussion and conclusion}

The results presented in Figs. 3 and 4 show good agreement with the theoretical predictions of Herterich and Hasselmann (1982), particularly at the lowest values of the particle separation. These results are for a wind speed of $10 \mathrm{~m} \mathrm{~s}^{-1}$. For any surface spectrum with the form of

Eq. (2), $D_{i j}$ can be written

$D_{i j}=\alpha^{2} g^{4} \omega_{\mathrm{m}}^{-3} \overline{D_{i j}}$

where $\overline{D_{i j}}$ is a non-dimensional function of $S$ and $\alpha=0.0081$ is the Phillips constant (Herterich and Hasselmann 1982). For the Pierson-Moskowitz spectrum, the peak frequency $\omega_{\mathrm{m}}$ is given by Eq. (6). This means that, once the surface diffusion coefficients have been calculated for one velocity for a given $S$ and $\psi$, they can be found for any other velocity using the scaling relationship

$D_{i j}\left(U_{1}\right)=\left(\frac{U_{2}}{U_{1}}\right)^{3} D_{i j}\left(U_{2}\right)$.

For a wind speed of $10 \mathrm{~m} \mathrm{~s}^{-1}$, the results show a significant diffusion rate, even at small particle separations of a tenth of the mean wavelength. As the wind speed increases, the diffusion tensor increases as $U^{3}$, producing a rapid increase in the rate at which diffusion occurs. An increase in the wind speed will, however, also increase the effect of other dispersive processes such as turbulence, which at high wind speeds, particularly if breaking occurs, will be the predominant means of diffusion. Thus, the importance of wave action in dispersing pollutants is most significant at low wind speeds (and length scales of up to a few kilometres). At higher wind speeds, where wave-induced diffusion is greater, other forms of diffusion are predominant, however, the wave diffusion need not be negligible and so should be considered in any accurate model. This is the case if the region being considered is less than a few square kilometres, such as an estuary of harbour area.

In conclusion, the diffusion of tracers on a random sea surface was considered. Experiments were performed in a three-dimensional basin and the diffusion coefficient found for particles on the surface. The results showed good agreement with existing theory.

\section{References}

Craig PD; Banner ML (1994) Modelling wave-enhanced turbulence in the ocean surface layer. J Phys Oceanogr 24: 2546-2559

Herterich K; Hasselmann K (1982) The horizontal diffusion of tracers by surface waves. J Phys Oceanogr 12: 704-711

Maksimenko NA (1991) Comparative analysis of Lagrangian statistical characteristics for synoptic-scale currents in hydrophysical study areas. Oceanology 30: 5-9

Mesquita ON; Kane S; Gollub JP (1992) Transport by capillary waves: fluctuating Stokes drift. Phys Rev A45: 3700-3705

Pierson WJ; Moskowitz L (1964) A proposed spectral form for fully developed wind seas. J Geophys Res 69: 5181-5190

Sanderson BG; Okubo A (1988) Diffusion by internal waves. J Geophys Res 93: 3570-3582

Sanderson BG; Pal BK (1990) Patch diffusion computed from Lagrangian data, with application to the Atlantic equatorial undercurrent. Atmos Ocean 28: 444-465

Schott F; Ehlers M; Hubrich L; Quadfasel D (1978) Small-scale diffusion experiments in the Baltic surface-mixed layer under different weather conditions. Deutsch Hydrogr Z 31: 195-215 